\title{
Music as Mathematics of Senses
}

\author{
Hailong Li' ${ }^{1}$, Kalyan Chakraborty ${ }^{2}$, Shigeru Kanemitsu ${ }^{3}$ \\ ${ }^{1}$ Department of Mathematics, Weinan Normal University, Weinan, China \\ ${ }^{2}$ Harish-Chandra Research Institute, Allahabad, India \\ ${ }^{3}$ Faculty of Engineering, Kyushu Institute of Technology, Kitakyushu, Japan \\ Email: lihailong@wntc.edu.cn, kalyan@hri.res.in, omnikanemitsu@yahoo.com
}

How to cite this paper: Li, H.L., Chakraborty, K. and Kanemitsu, S. (2018) Music as Mathematics of Senses. Advances in Pure Mathematics, 8, 845-862. https://doi.org/10.4236/apm.2018.812052

Received: August 31, 2018

Accepted: December 18, 2018

Published: December 21, 2018

Copyright $\odot 2018$ by authors and Scientific Research Publishing Inc. This work is licensed under the Creative Commons Attribution International License (CC BY 4.0).

http://creativecommons.org/licenses/by/4.0/

\section{(c) (i) Open Access}

\begin{abstract}
It is often said that music has reached its supreme and highest level in the 18 th and 19th centuries. One of the main reasons for this achievement seems to be the robust structure of compositions of music, somewhat remindful of robust structure of mathematics. One is reminded of the words of Goethe: Geometry is frozen music. Here, we may extend geometry to mathematics. For the Middle Age in Europe, there were seven main subjects in the universities or in higher education. They were grammar, logic and rhetoric-these three (tri) were regarded as more standard and called trivia (trivium), the origin of the word trivial. And the remaining four were arithmetic, geometry, astronomy and music - these four (quadrus) were regarded as more advanced subjects and were called quadrivia (quadrivium). Thus for Goethe, geometry and mathematics seem to be equivocal. G. Leibniz expresses more in detail in his letter to C. Goldbach in 1712 (April 17): Musica est exercitium arithmeticae occultum nescientis se numerari animi (Music is a hidden arithmetic exercise of the soul, which doesn't know that it is counting). Or in other respects, J. Sylvester expresses more in detail: Music is mathematics of senses. Mathematics is music of reasons. Thus, the title arises. This paper is a sequel to [1] and examines mathematical structure of musical scales entailing their harmony on expanding and elaborating material in [2] [3] [4] [5], etc. In statistics, the strong law of large numbers is well-known which claims that $P\left\{\lim _{n \rightarrow \infty} \frac{r}{n}\right\}=1$. This means that the relative frequency $\frac{r}{n}$ of occurrences of
\end{abstract} an event $A$ tends to the true probability $p$ of the occurrences of $A$ with probability 1 . In music, harmony is achieved according to Pythagoras' law of small numbers, which claims that only the small integer multiples of the fundamental notes can create harmony and consonance. We shall also mention the law of cyclotomic numbers according to Coxeter, which elaborates Pythagoras' law and suggests a connection with construction of $n$-gons by ruler and compass. In the case of natural scales (just intonation), musical notes appear 
in the form $2^{p} 3^{q} 5^{r} \quad$ (multiples of the basic note), where $p \in \mathbb{Z}$, $q=-3,-2,-1,0,1,2,3$ and $r=-1,0,1$. We shall give mathematical details of the structure of various scales.

\section{Keywords}

Pythagorean Scale, Just Intonation, Temperament, Beat, Pythagoras' Law of Small Numbers, Law of Cyclotomic Numbers

\section{Distance Functions}

The pitch of a musical note (hereafter abbreviated as a note) is defined by its frequency measured in Herz $(\mathrm{Hz})$, cycles per second.

The bigger the frequency, the higher the pitch.

The frequencies of all musical notes are the set of positive reals $\mathbb{R}_{+}$. The interval between them is expressed by the ratio of their frequencies. As will be shown below, in just intonation, $D=\frac{9}{8}$ and $A=\frac{5}{3}$ and the interval between them are calculated by division $\frac{5}{3} / \frac{9}{8}=\frac{40}{27}$. The reason for this is that use is made of the logarithmic distance and the subtraction of logarithms is expressed as division of the anti-logs. In turn, the main reason why the logarithmic distance is used comes from the Weber-Fechner law (cf. e.g. [6]) to the effect that stimuli are sensed logarithmically. For two notes $a$ and $b$ define

$$
d(a, b)=|\log a / b|=|\log a-\log b| \geq 0 .
$$

Then this is a distance function. For $d(a, a)=0, d(b, a)=d(a, b)$ and

$$
\begin{aligned}
d(a, c) & =|\log a-\log b+\log b-\log c| \\
& \leq|\log a-\log b|+|\log b-\log c| \\
& =d(a, b)+d(b, c) .
\end{aligned}
$$

Hence, $\left(\mathbb{R}_{+}, d\right)$ is a metric space. Thus, we think of intervals between two notes as the logarithmic difference, which we substitute by the quotient, say $b / a$, of corresponding frequencies $b>a$ and say that the interval between $a$ and $b$ is $b / a$ (by which we understand the logarithmic interval $\log b-\log a$ ).

\section{Equivalence Classes}

We introduce the relation $\sim$ by

$$
a \sim b \Leftrightarrow a / b=2^{n} \quad n \in \mathbb{Z} .
$$

Then this is an equivalence relation. For $a / a=2^{0}$, and if $a / b=2^{n}$, then $b / a=2^{-n}$. Finally, if $a / b=2^{m}$ and $b / c=2^{n}$, then $a / c=2^{m+n}$. Hence $\mathbb{R}_{+}$is classified into equivalence classes: $\mathbb{R}_{+} / \sim$.

This may be also viewed group-theoretically. The multiplicative group $\mathbb{R}_{+}$or its subgroup $\mathbb{Q}_{+}$of all positive rationals contains the cyclic subgroup 
$\langle 2\rangle=\left\{2^{n} \mid n \in \mathbb{Z}\right\}$. And the quotient group $\mathbb{Q}_{+} /\langle 2\rangle$ may be considered as the set of all musical notes up to octaves.

Two musical notes whose frequencies are different by powers of 2 are thought of as belonging to the same equivalence class, or those which sound alike. Musicians express the equivalence relation by using the same label for those notes in the same class. E.g. if the middle $C$ has frequency $260 \mathrm{~Hz}$, then the note three octaves higher than middle $C$ has frequency $260 \times 2^{3}=2080 \mathrm{~Hz}$ while the note two octaves below middle $C$ has frequency $260 \times 2^{-2} \approx 65 \mathrm{~Hz}$. They are denoted by the same symbol $C$ and are referred to high $C$ or low $C$, respectively. The concert pitch which is used today is $A=440$. Then with this value, in Pythagoras scale, the middle $\mathrm{C}=261$ while in just intonation it is $\mathrm{C}=264$, cf. also (6.1).

Definition 1. Two intervals which combine to give an octave is called an inversion to each other.

After the octave, the next simplest is the perfect fifth 3:2 containing 7 semitones whose inversion is the perfect fourth 4:3 containing 5 semitones. The major third 5:4 is the interval containing 4 semitones whose inversion is the minor sixth 8:5 which contains 8 semitones (Figure 1 \& Figure 2).

The sequence of three notes arranged in the order of the major third and the minor third is called a major triad.

The common major chord (do-mi-so-do) has the ratio 4:5:6:8, while the common minor chord (do-mí-so-do) has the ratio 10:12:15:20 (Figure 3).

Remark 2.1 For the meaning of "fifth', cf. Remark 5.1 below. There are three ratios with the prefix "perfect', which are perfect first (which is nothing but the unison), perfect fifth and perfect fourth. The major third is the interval from $A(f=440)$ to $C^{\#}(f=550)$, which is a representative of the equivalence class consisting of the intervals $5 / 4,5 / 2,5 / 1,10 / 1, \ldots$

\section{Pythagoras Law of Small Numbers and the Helmholtz-Joahim Scale}

Pythagoras was the first who raised the question "Why is consonance associated with the ratios of small numbers?" Here "numbers" means integers. This is referred to as Pythagoras' law of small numbers to the effect that only the small integer multiples of the fundamental notes can create harmony and consonance. There is a variation of this law, the law of cyclotomic numbers, cf. $\S 7$.

In this section as a partial explanation of Pythagoras' law of small numbers, we refer to the experiment of Helmholtz and Joahim stated in [3] and we refer to

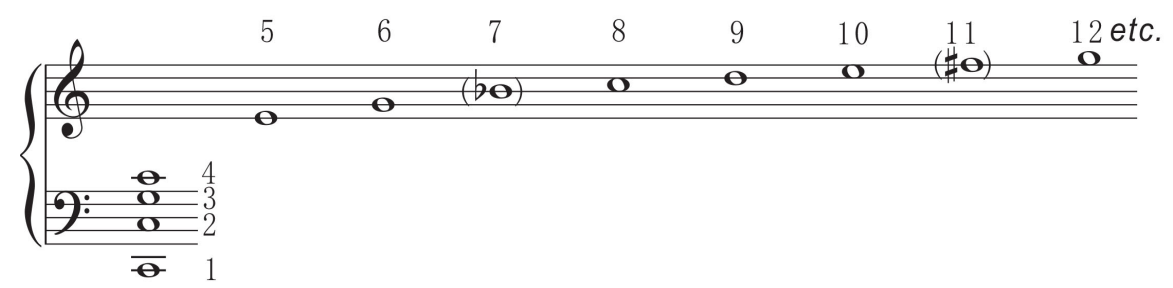

Figure 1. Natural harmonic series. 


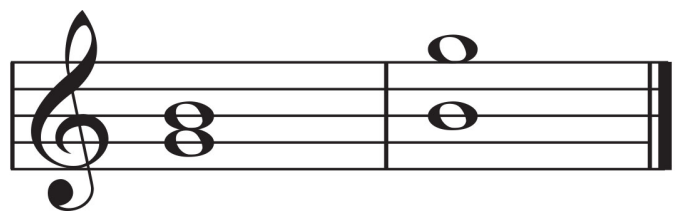

Figure 2. Inversions.

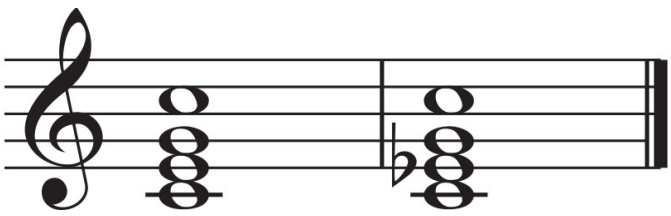

Figure 3. Common chords.

their result as the Helmhotz-Joahim scale. H. von Helmholtz with the help of the renowned violinist J. Joachim, made an experiment and tabulated the notes which are the most pleasing to the ears (Table 1).

Table 2 is the basis of Coxeter's speculation of the law of cyclotomic numbers in $\S 7$.

\section{Various Scales}

When we pile up the notes starting from a basic note, we obtain an increasing sequence of pitches. The way of piling up is not at one's disposal and one has to struggle to achieve good consonants among them. There are several sequences constructed and they are called a scale or sometimes pitch with the inventor's name or with the describing term (sometimes also called an intonation).

In the case of Pythagorean scale ( $\S 5$ ), it is formed using only powers of 2 and 3.

Since one whole-tone consists of two semi-tones and there are two semi-tones and five whole-tones in one octave, it follows that one octave has 12 semitones, cf. (8.6) below. Therefore, piling up the notes on the basic one, the 12th power is very important. In the case of the Pythagorean scale, what are piled up are powers of $\frac{3}{2}$, so that

$$
\left(\frac{3}{2}\right)^{12} \approx 129.7
$$

which is a little higher than 7 octaves: $2^{7}=128$. The interval

$$
\frac{129.7}{128}
$$

is known as the comma of Pythagoras. This discrepancy accounts for many difficulties in obtaining an organized system of pitches.

In the equal tempered system, one octave is equally divided into 12 semitones with ratio being the 12th root of unity

$$
1 \text { tempered semitone }=2^{1 / 12}=1.05946 \cdots
$$


Table 1. (a) Helmholtz-Joachim scale. (b) Helmholtz-Joachim scale (cont.).

(a)

\begin{tabular}{ccccccc}
\hline & C & Eb & E & F & G & Ab \\
int. & unison & minor 3rd & major 3rd & perfect 4th & perfect 5th & minor 6th \\
ratio & $\frac{1}{1}$ & $\frac{6}{5}$ & $\frac{5}{4}$ & $\frac{4}{3}$ & $\frac{3}{2}$ & $\frac{8}{5}$ \\
note & do & mi\# & mi & fa & sol & la\# \\
\hline
\end{tabular}

(b)

\begin{tabular}{ccc}
\hline from C & A & C \\
\hline interval & major sixth & octave \\
ratio & $\frac{5}{3}$ & $\frac{2}{1}$ \\
note & la & do \\
\hline
\end{tabular}

Table 2. Multiples of low C.

\begin{tabular}{lllllllllllll}
\hline mult. & 1 & 2 & 3 & 4 & 5 & 6 & 7 & 8 & 9 & 10 & 11 & 12 \\
note & $\mathrm{C}$ & $\mathrm{C}$ & $\mathrm{G}$ & $\mathrm{C}$ & $\mathrm{E}$ & $\mathrm{G}$ & - & $\mathrm{C}$ & $\mathrm{D}$ & $\mathrm{E}$ & - & $\mathrm{G}$ \\
\hline
\end{tabular}

\section{The Pythagorean Scale}

The Pythagorean scale is made of $2^{p} 3^{q}, p, q \in \mathbb{Z},-3 \leq q \leq 3$. As is explained above, starting from middle C, A (la) is obtained as $\left(\frac{3}{2}\right)^{3} \sim \frac{27}{16}$.

From middle $C\left(\right.$ doh) by piling up $\frac{3}{2}$, we get $G\left(\right.$ sol), then $\left(\frac{3}{2}\right)^{2}=\frac{9}{4} \sim \frac{9}{8}$, which is $\mathrm{D}(\mathrm{re})$. The next is $\left(\frac{3}{2}\right)^{3}=\frac{27}{8} \sim \frac{27}{16}$, which is A (la) with frequency $440 \mathrm{~Hz}$. The 5th is $\left(\frac{3}{2}\right)^{4}=\frac{81}{16} \sim \frac{81}{64}=\left(\frac{3}{2}\right)^{2}$, which is $\mathrm{E}(\mathrm{mi})$.

Definition 2. These 5 notes $C, D, E, A, G$ form the pentatonic scale. It is the basis of many folk-songs in Scotland. Figure 4.

The 6th is $\left(\frac{3}{2}\right)^{5}=\frac{243}{32} \sim \frac{243}{32} \frac{1}{2^{2}}=\frac{243}{128}$, which is B (si). Thus C (doh), G (sol), D (re), A (la), E (me), B (si). The 7th F (fa) is obtained by coming back from higher C. i.e. $2 \cdot\left(\frac{3}{2}\right)^{-1}=\frac{3}{2}$.

This is the scale used by the Greeks and early medieval composers as the basis of the Ecclesiastic Mode. The scale is suitable for melodic writing but not satisfactory for harmonic writing (for modulation) (Figure 5).

We remark that the sequence $\left\{\left[\left(\frac{3}{2}\right)^{n}\right]\right\}$ is very much relevant to number theory, esp. in connection with the Waring problem, cf. e.g. [7], where the Gaussian symbol will be defined after (5.1). 


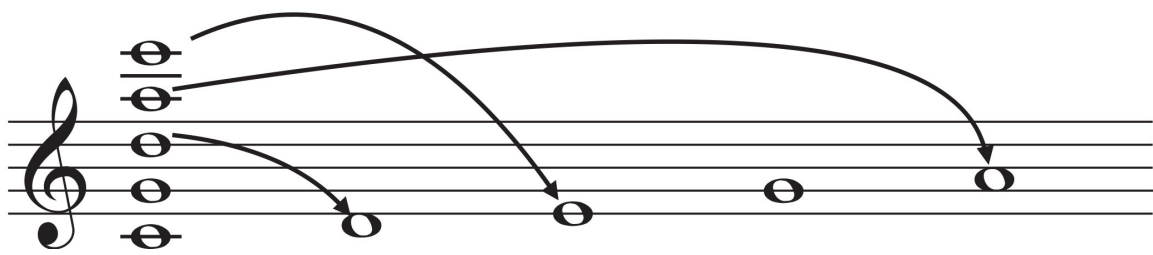

Figure 4. Pentatonic scale.

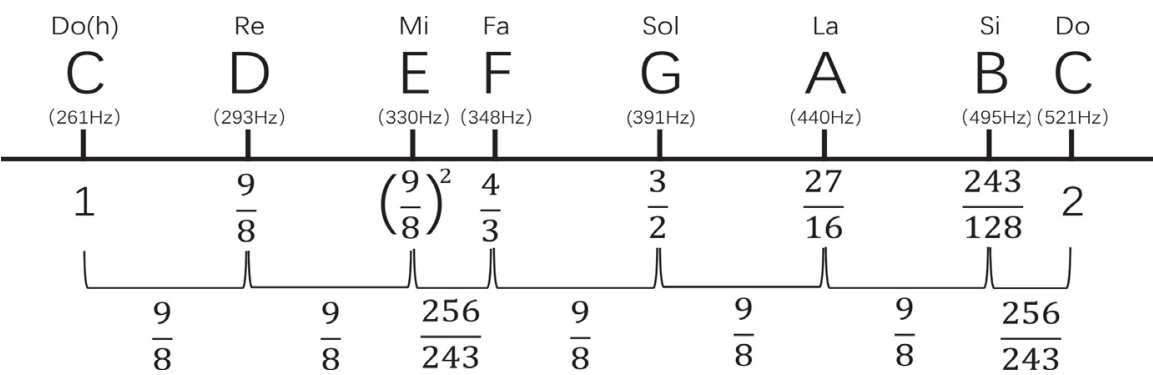

Figure 5. The Pythagorean Scale.

\subsection{Major and Minor Chords}

The home key could be any one of seven notes but what survived in tone-centered music nowadays are the Ionian scale beginning and ending on $\mathrm{C}$ and the Aeolian scale (with key-note A). The Ionian and Aeolian scales are known as the ordinary major and minor scales (Table 3 ).

Definition 3. The major triad is the piling up of the major third followed by the perfect fifth on the root. The minor triad is the piling up of the minor third followed by the perfect fifth on the root (Figure 6).

On [2] there are two speculations for the reason why those two chords remain. One is that the interval from si to do is very small and gives the impression that it is coming to an end. The other is more convincing that as one can see, the major triads are situated symmetrically over the octave, and so these chords survived. But although in the figure it is apparent, it is not certain whether one senses this symmetry by ears. We guess that this is not a reasoning by senses but by reasons. On [2], it is stated that Bach's music is quite mathematical but for Bach, music comes first and the accompanying mathematical structure is rather the by-products of composing music-as mathematics of senses. [8] [9] [10] [11] etc. on Bach's music and its plausible relation with mathematics.

\subsection{Arithmetic of the Pythagorean Scale}

In $\S 5$, the piling up method is given of constructing Pythagorean scale, but each time one has to think which power $p$ of $2^{-1}$ is to be multiplied to shift the note into the interval $[1,2]$. In this section we show the following theorem which gives the value of the exponent $p$ of 2 uniquely, given $q$ of the exponent of 3. Here we extend the range of $q$ to $|q| \leq 6$. We appeal to the numerical values

$$
\log 2=0.69315, \quad \log 3=1.09861, \quad \alpha=\frac{\log 3}{\log 2},
$$


Table 3. The Ionian and Aeolian scales are known as the ordinary major and minor scales.

\begin{tabular}{ccc}
\hline & begin & key-note \\
\hline Ionian & $\mathrm{C}$ & major \\
Aeolian & $\mathrm{A}$ & minor \\
\hline
\end{tabular}

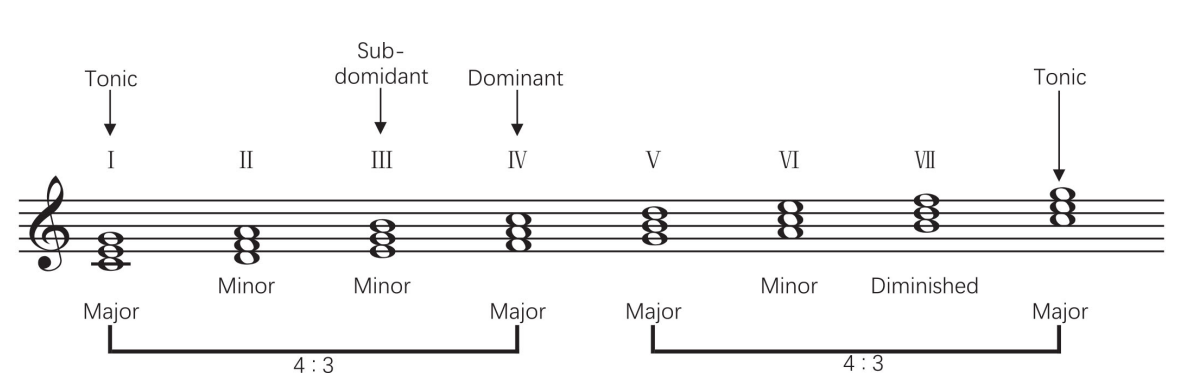

Figure 6. The position of major triads.

where the logarithm means the natural logarithm.

Theorem 5.1 1) The unique integer $p$ satisfying the conditions

$$
1<2^{p} 3^{q} \leq 2, \quad|q| \leq 5
$$

is $p=[-\alpha q]+1$, where the symbol $[x]$ indicates the integral part (the Gauss symbol) of the real number $x$.

2) For an integer $p$ to exist that satisfies

$$
1<2^{p} 3^{q} \leq \frac{3}{2}, \quad|q| \leq 4
$$

the inequality

$$
\{-\alpha q\}>2-\alpha=1.42 \cdots
$$

must hold. Cf. Table 4 \& Table 5.

Proof. The restriction (5.1) amounts to

$$
-\alpha q<p \leq-\alpha q+1,
$$

and the interval $(-\alpha q,-\alpha q+1)$ has length exactly 1 , so that it contains a unique integer, which is $p=[-\alpha q]+1$. (5.3) follows similarly.

Remark 5.1. 1) We note that the value $\frac{729}{512}$ for Fis invalidates the law of small numbers in $\S 3$. In order to overcome this difficulty, we choose the fraction $x$ such that $\frac{243}{128} \cdot \mathrm{F}=\frac{729}{512}$, i.e. $\mathrm{F}=\frac{4}{3}$. Then this awkward number appears as the distance between $\mathrm{B}$ and $\mathrm{F}: \mathrm{B} \div \mathrm{F}=\frac{243}{128} \times \frac{3}{4}=\frac{729}{512}$. Also the fractions $\frac{16}{9}$, $\frac{32}{27}, \frac{128}{81}$ appear as the difference between $D$ and (high) $C, F$ and $D$, and $E$ and C.

2) From Figure 5.1 we see that there are 5 tones under $G$ including $G$ itself. $C$, $D, E, F, G$, thus justifying the name " 5 th" in Definition 1. 2) The semi-tone $\tilde{s}$ and\# in Definition 4 are called limma and apotome, respectively. 
Table 4. Values associated to Theorem 5.1.

\begin{tabular}{cccccc}
\hline value of $q$ & $-\alpha q$ & $\{-\alpha q\}$ & $p$ & $2^{p} 3^{q}$ & note \\
\hline$q=1$ & -1.58495 & $0.42 \cdots$ & $p=-1$ & $\frac{3}{2}$ & $\mathrm{G}$ \\
$q=2$ & $-3.16 \cdots$ & $0.94 \cdots$ & $p=-3$ & $\frac{9}{8}$ & $\mathrm{D}$ \\
$q=3$ & $-4.74 \cdots$ & $0.26 \cdots$ & $p=-4$ & $\frac{27}{16}$ & $\mathrm{~A}$ \\
$q=4$ & $-6.32 \cdots$ & $0.68 \cdots$ & $p=-6$ & $\left(\frac{9}{8}\right)^{2}$ & $\mathrm{E}$ \\
$q=5$ & $-7.90 \cdots$ & $0.10 \cdots$ & $p=-7$ & $\frac{243}{128}$ & $\mathrm{~B}$ \\
$q=6$ & $-9.50 \cdots$ & $0.50 \cdots$ & $p=-9$ & $\frac{1}{8}\left(\frac{3}{2}\right)^{6}=\frac{729}{512}$ & $\mathrm{Fis}$ \\
$q=7$ & $-11.06 \cdots$ & $0.50 \cdots$ & $p=-11$ & $\frac{2187}{2048}$ & \# \\
$q=-1$ & $1.58 \cdots$ & $0.58 \cdots$ & $p=2$ & $\frac{4}{3}$ & $\mathrm{~F}$ \\
$q=-2$ & $3.16 \cdots$ & $0.16 \cdots$ & $p=4$ & $\frac{16}{9}$ & $\mathrm{D}-\mathrm{C}$ \\
$q=-3$ & $4.74 \cdots$ & $0.74 \cdots$ & $p=5$ & $\frac{32}{27}$ & $\mathrm{D}-\mathrm{F}$ \\
$q=-4$ & $6.32 \cdots$ & $0.32 \cdots$ & $p=7$ & $\frac{128}{81}$ & $\mathrm{E}-\mathrm{C}$ \\
\hline & $7.90 \cdots$ & $0.90 \cdots$ & $p=8$ & $\frac{256}{243}$ & $\tilde{s}$ \\
\hline
\end{tabular}

Table 5. Frequency ratios in Pythagorean scale.

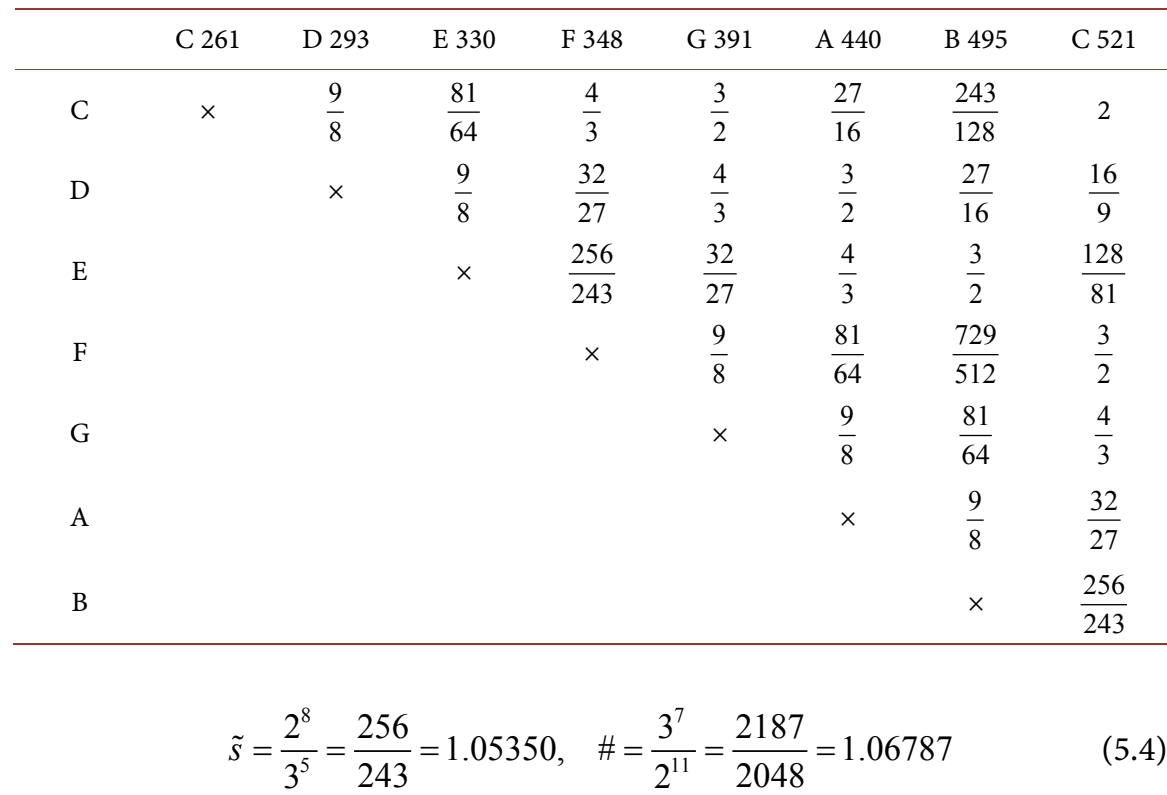

\section{The Verdi Pitch}

The Verdi pitch is the scale which fixes the freq. of A to be 432. In [6] the following theorem has been found. 
Theorem 6.1 The reason for the $A=432$ is that in the Pythagorean scale,

$$
\mathrm{C}=432 \times\left(\frac{27}{16}\right)^{-1}=256=2^{8} .
$$

This makes some of the calculations simpler but this was not used much. The reason is that the instruments are tuned not from $\mathrm{C}$ but from $\mathrm{A}$ and in just intonation it gives a fractional frequency to $\mathrm{A}: \mathrm{A}=256 \times \frac{5}{3}=426 \frac{3}{2} \approx 427$. This is approximately the standard pitch used in the time of Beethoven (Figure 7).

\section{The Just Intonation and the Law of Cyclotomic Numbers}

The Pythagorean major 3rd $m=\frac{81}{64}$ is slightly bigger than the major $3 \mathrm{rd} \frac{5}{4}$ of just intonation. The Pythagorean major 3rd is said to let the melody sound beautifully, but it diminishes harmony because of the beats contained. In $\{d, m\}$ the number of beats caused by the 4 times and 5 times of $m$ is

$$
d\left(4 \times \frac{81}{64}-5 \times 1\right)=\frac{d}{16}=0.0625 d,
$$

which is 16.5 times/s for $d=264 \mathrm{~Hz}$, say. Hence in the Pythagorean major triad

$$
d\left\{1, \frac{m}{d}, \frac{s}{d}\right\}=d\left\{1, \frac{\mathrm{q}^{4}}{4}, \mathrm{q}\right\}=d\left\{1, \frac{81}{64}, \frac{3}{2}\right\},
$$

there occur beats between the root and the major 3rd. To eliminate this beat, we decrease the major third by multiplying by the syntonic comma

$$
\frac{1}{\Delta}=\frac{80}{81}
$$

to make it $m=\frac{5}{4}$. This yields the just intonation, in which the major $3 \mathrm{rd}$ consists of

$$
\left\{1, \frac{m}{d}, \frac{s}{d}\right\}=\left\{1, \frac{\mathrm{q}^{4} \Delta}{4}, \mathrm{q}\right\}=\left\{1, \frac{5}{4}, \frac{3}{2}\right\}=\frac{1}{4}\{4,5,6\} .
$$

Figure 8 and Table 6.

On the basis of Table 2, Coxeter [3] states a speculation that the agreeable harmonics

$$
3,4,5,6,8,10,12
$$

correspond to the number of sides of regular polygons that can be constructed by a ruler and a compass. A natural inverse question is where there are corresponding harmonics to 15 and 17 since 15-gon was constructed by Euclid while 17-gon was by Gauss in 1797. The interval 15 from a low C to high B which thrilled the audience appears as the appoggiatura in the end of St. Matthew Passion (Figure 9). The interval 17 were used by the remaining two of the 3 B's, i.e. by Beethoven and Brahms. Since regular polygons inscribed in a circle divides it into equal parts, cyclotomy, Coxeter refers to the law of cyclotomic numbers rather than the law of small numbers. 


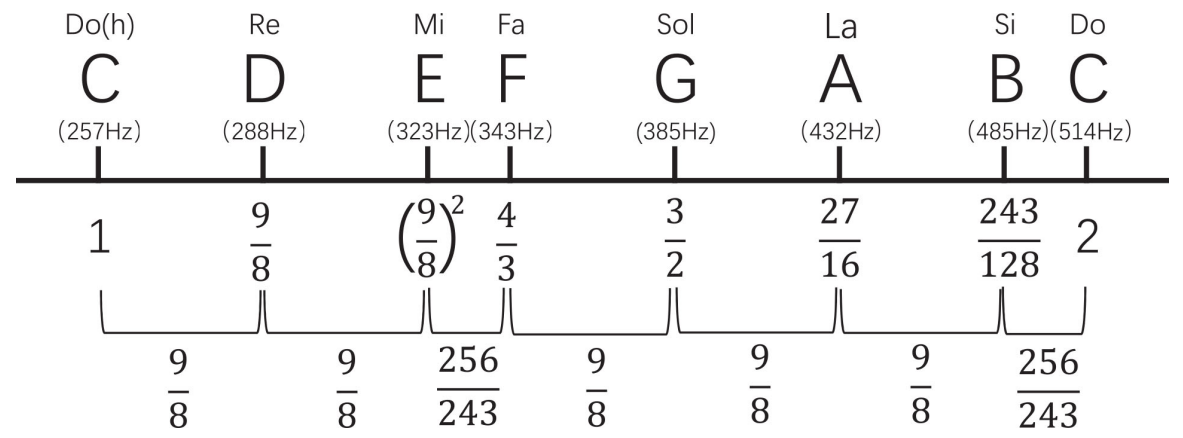

Figure 7. The Verdi pitch.

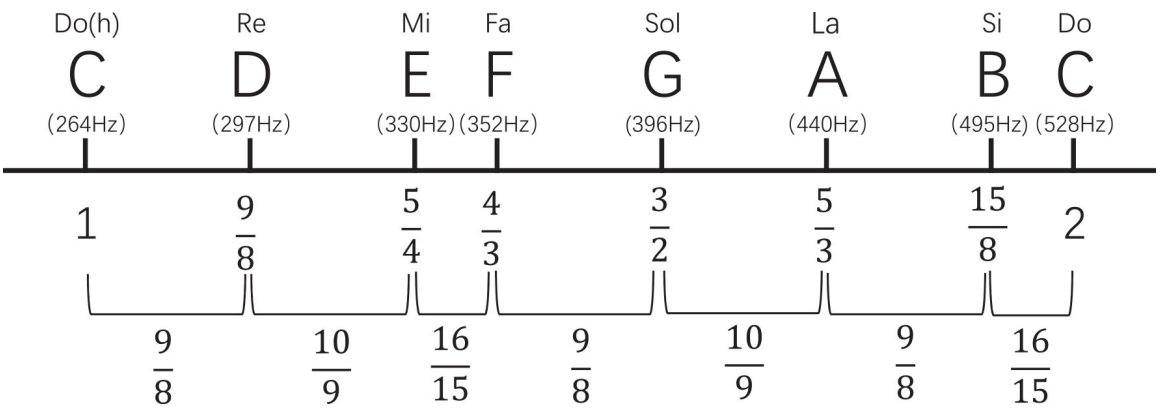

Figure 8. Just intonation scale.

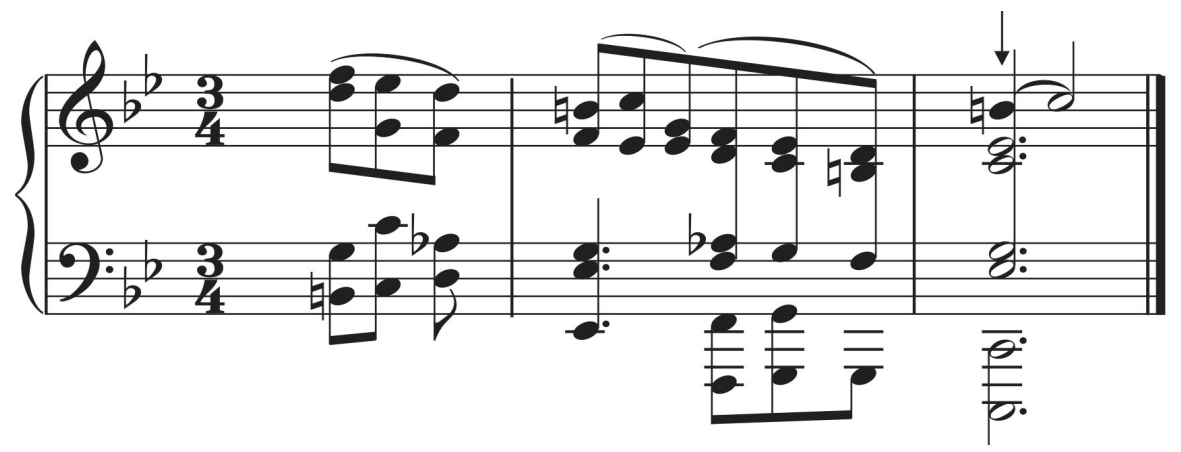

Figure 9. Appoggiatura at the end of St. Matthew Passion.

Table 6. Frequency ratios in just intonation scale.

\begin{tabular}{|c|c|c|c|c|c|c|c|c|}
\hline & C 264 & D 297 & E 330 & F 352 & G 396 & A 440 & B 495 & C 528 \\
\hline \multirow{2}{*}{ C } & $x$ & $\underline{9}$ & $\underline{5}$ & $\underline{4}$ & $\underline{3}$ & $\underline{5}$ & $\underline{15}$ & \multirow{2}{*}{2} \\
\hline & $x$ & $\overline{8}$ & $\overline{4}$ & $\overline{3}$ & $\overline{2}$ & $\overline{3}$ & $\overline{8}$ & \\
\hline \multirow{2}{*}{$\mathrm{D}$} & & \multirow{2}{*}{$x$} & $\underline{10}$ & 32 & $\underline{4}$ & $\underline{40}$ & $\underline{15}$ & $\underline{16}$ \\
\hline & & & 9 & 27 & 3 & $\overline{27}$ & $\overline{9}$ & $\overline{9}$ \\
\hline \multirow{2}{*}{ E } & & & \multirow{2}{*}{$x$} & 16 & $\underline{6}$ & $\underline{4}$ & $\underline{3}$ & 8 \\
\hline & & & & 15 & 5 & 3 & 2 & 5 \\
\hline \multirow{2}{*}{$\mathrm{F}$} & & & & $x$ & $\underline{9}$ & $\underline{5}$ & 45 & $\underline{3}$ \\
\hline & & & & $\lambda$ & $\overline{8}$ & 4 & 32 & 2 \\
\hline \multirow{2}{*}{ G } & & & & & $x$ & 10 & $\underline{5}$ & 4 \\
\hline & & & & & $\lambda$ & 9 & $\overline{4}$ & $\overline{3}$ \\
\hline \multirow{2}{*}{ A } & & & & & & $x$ & 9 & $\underline{6}$ \\
\hline & & & & & & $\lambda$ & $\overline{8}$ & 5 \\
\hline \multirow[t]{2}{*}{ B } & & & & & & & $x$ & 16 \\
\hline & & & & & & & & 15 \\
\hline
\end{tabular}




\section{Modulation}

Part of the contents of this section is an elaboration of material in [4]. In this section we use the notation $\{d, r, m, f, s, l, t, 2 d\}$, which are the initials in tonic sol-fa notation, doh, ray, me fah, soh, lah, te, doh. We also think of this as an increasing sequence of frequencies denoted by the corresponding symbols. We may conveniently write

$$
\{d, r, m, f, s, l, t, 2 d\}=d\left\{1, \frac{r}{d}, \frac{m}{d}, \frac{f}{d}, \frac{s}{d}, \frac{l}{d}, \frac{t}{d}, 2\right\}
$$

to express the ratios of each note against the basic $d$. We consider the modulation from $\mathrm{C}$ to $\mathrm{G}$. In order to express the correspondence, we expand (8.1) to obtain

$$
\begin{aligned}
F & =\{d, r, m, f, f \#, s, l, t, 2 d, 2 r, 2 m, 2 f, 2 f \#, 2 s\} \\
& =d\left\{1, \frac{r}{d}, \frac{m}{d}, \frac{f}{d}, \frac{f \#}{d} \frac{s}{d}, \frac{l}{d}, \frac{t}{d}, 2,2 \frac{r}{d}, 2 \frac{m}{d}, 2 \frac{f}{d}, 2 \frac{f \#}{d}, 2 \frac{s}{d}\right\} .
\end{aligned}
$$

To modulate $\mathrm{C}$ major into $\mathrm{G}$ major is just to replace the basic note $d$ in (8.2) by $s$ by which we obtain the sequence, thereby adding the prime to indicate the correspondence

$$
\begin{aligned}
& \left\{f^{\prime}, s^{\prime}, l^{\prime}, t^{\prime}, d^{\prime}, r^{\prime}, m^{\prime}, f^{\prime}, s^{\prime}, l^{\prime}, t^{\prime}, d^{\prime}\right\} \\
& =s\left\{\frac{f^{\prime}}{s}, \frac{s^{\prime}}{s}, \frac{l^{\prime}}{s}, \frac{t^{\prime}}{s} \frac{d^{\prime}}{s}, \frac{r^{\prime}}{s}, \frac{m^{\prime}}{s}, 2, \frac{f^{\prime}}{s}, \frac{s^{\prime}}{s}, \frac{l^{\prime}}{s}, \frac{t^{\prime}}{s}, 2 \frac{d^{\prime}}{s}\right\},
\end{aligned}
$$

where we used the relation $d^{\prime}=s$. In general by comparing the frequencies, we obtain

$$
d^{\prime}=s, r^{\prime}=l, m^{\prime}=t, f^{\prime}=2 d, s^{\prime}=2 r, l^{\prime}=2 m, t^{\prime}=2 f \# .
$$

Also comparing the ratios in (8.2) and (8.3), we find that the ratios with prime are equal to those without:

$$
\frac{x^{\prime}}{s}=\frac{x^{\prime}}{d^{\prime}}=\frac{x}{d}
$$

for all $x \in F$, e.g. $\frac{s^{\prime}}{s}=\frac{s^{\prime}}{d^{\prime}}=\frac{s}{d}$, the far-right side member we denote by $q$.

$$
\mathrm{q}=\frac{s}{d}
$$

Then (8.5) amounts to

$$
x^{\prime}=\mathrm{q} x \text {. }
$$

With $d$ and $q$ as parameters, we may express all other notes in terms of them. First $s=\mathrm{q} d$. Using (8.4) and (8.5), we have $2 r=s^{\prime}=\mathrm{q} s$, whence $r=\frac{1}{2} \mathrm{q}^{2} d$. Similarly, from $2 d=f^{\prime}=\mathrm{q} f$ we have $f=\frac{2}{\mathrm{q}} \mathrm{q} d$. Since $l=r^{\prime}=\mathrm{q} r$ we deduce that $l=\mathrm{q} \frac{1}{2} \mathrm{q}^{2} d=\frac{\mathrm{q}^{3}}{2} d \quad$. From $2 m=l^{\prime}=\mathrm{q} l \quad$ we deduce that $m=\mathrm{q} \frac{1}{2} \mathrm{q}^{3} d=\frac{\mathrm{q}^{4}}{4} d$. From $t=m^{\prime}=\mathrm{q} m$, it follows that $t=\frac{\mathrm{q}^{5}}{4} d$. Finally, from 
$2 \frac{2}{\mathrm{q}} d \#=2 f \#=t^{\prime}=\mathrm{q} t=\frac{\mathrm{q}^{6}}{4} d$, it follows that $\#=\frac{\mathrm{q}^{7}}{16}$.

Theorem 8.1 The major chord in Table 3 is expressed as

$$
\begin{aligned}
F & =\{d, r, m, f, s, l, t, 2 d\} \\
& =d\left\{1, \frac{\mathrm{q}^{2}}{2}, \frac{\mathrm{q}^{4}}{4}, \frac{2}{\mathrm{q}}, \mathrm{q}, \frac{\mathrm{q}^{3}}{2}, \frac{\mathrm{q}^{5}}{4}, 2\right\}
\end{aligned}
$$

while the minor chord as

$$
\begin{aligned}
F & =\{l, t, d, r, m, f, s, 2 l\} \\
& =l\left\{1, \frac{\mathrm{q}^{2}}{2}, \frac{4}{\mathrm{q}^{3}}, \frac{2}{\mathrm{q}}, \mathrm{q}, \frac{8}{\mathrm{q}^{4}}, \frac{4}{\mathrm{q}^{2}}, 2\right\} .
\end{aligned}
$$

Corollary 8.1 The Pythagorean scale is the case with $\mathrm{q}=\frac{3}{2}$.

Remark 8.1 Although Theorem 8.1 explains the reason why fis chosen as $\frac{2}{\mathrm{q}}$, it is a typical hindsight since Pythagoras had no concept of modulation and he must have followed the reasoning given in Remark 5.1, 1) based on the law of small numbers.

Corollary 8.2 There are three major triads, $\left\{1, \frac{\mathrm{q}^{4}}{4}, \mathrm{q}\right\}=\{d, m, s\}=\mathrm{CEG}$, $\{f, l, d\}=\mathrm{FAC}, \quad\{s, t, r\}=\mathrm{GBD}$.

Proof. There are three major thirds $\frac{\mathrm{q}^{4}}{4}=\left\{\frac{m}{d}, \frac{l}{f}, \frac{t}{s}\right\}$ and so the piling up of minor thirds are possible only for those starting with $m, l, t$.

Definition 4 The major $2 n d$ is usually called tome and denoted by $w$ and the minor 2 nd is called semi-tome denoted $\tilde{s}$ :

$$
w=\frac{\mathrm{q}^{2}}{2}, \quad \tilde{s}=\frac{8}{\mathrm{q}^{5}} .
$$

1 octave consists of 5 tones and 2 semi-tones:

$$
\frac{2 d}{d}=\frac{r}{d} \frac{m}{r} \frac{f}{m} \frac{s}{f} \frac{l}{s} \frac{t}{l} \frac{2 d}{t}=w w \tilde{s} w w w \tilde{s}=w^{5} \tilde{s}^{2}
$$

in conformity with the statement in $\S 4$. Table 7 .

\section{Equal Temperament}

The law of cyclotomic numbers work well within the same key, but produces a contradiction if we modulate into other keys. In Table 6, the whole tone from $\mathrm{E} b$ to $F$ or from $G$ to $A$ is $\frac{4}{3} \frac{5}{6}=\frac{10}{9}$ while for the tone from $F$ to $G$ is $\frac{3}{2} \frac{3}{4}=\frac{9}{8}$ and they are different by $\frac{9}{8} \frac{9}{10}=\frac{81}{80}$, which the Greeks called a comma. To overcome this difficulty, the well-tempered scale was invented shortly before the time of Bach (Figure 10). Bach composed "Well-tempered Clavier". a masterpiece 
Table 7. Full range of musical intervals.

\begin{tabular}{|c|c|c|c|c|}
\hline name & ratio & nat. & temp. & semi-tones \\
\hline unison & $\frac{d}{d}$ & $\frac{1}{1}$ & 1.0000 & 1 \\
\hline octave & $\frac{2 d}{d}$ & $\frac{2}{1}$ & 2.0000 & 12 \\
\hline perfect 5 th & $\frac{s}{d}=\frac{l}{r}=\cdots=\frac{2 m}{l}=\mathrm{q}$ & $\frac{3}{2}$ & 1.4983 & 7 \\
\hline perfect 4 th & $\frac{f}{d}=\frac{s}{r}=\cdots=\frac{2 m}{t}=\mathrm{q}$ & $\frac{4}{3}$ & 1.3348 & 5 \\
\hline major 3rd & $\frac{m}{d}=\frac{l}{f}=\frac{t}{s}=\frac{\mathrm{q}^{4}}{4}$ & $\frac{5}{4}$ & 1.2599 & 4 \\
\hline minor 3rd & $\frac{f}{r}=\frac{s}{m}=\frac{2 d}{l}=\frac{2 r}{t}=\frac{4}{\mathrm{q}^{3}}$ & $\frac{6}{5}$ & 1.1892 & 3 \\
\hline major 6th & $\frac{l}{d}=\frac{t}{r}=\frac{2 r}{f}=\frac{2 m}{s}=\frac{\mathrm{q}^{3}}{2}$ & $\frac{5}{3}$ & 1.6818 & 9 \\
\hline minor 6 th & $\frac{m}{d}=\frac{l}{f}=\frac{t}{s}=\frac{8}{\mathrm{q}^{4}}$ & $\frac{8}{5}$ & 1.5874 & 8 \\
\hline major 2 nd & $\frac{r}{d}=\frac{m}{r}=\frac{s}{f}=\frac{l}{s}=\frac{t}{l}=\frac{\mathrm{q}^{2}}{2}$ & $\frac{9}{8}$ & 1.1225 & 2 \\
\hline minor 7 th & $\frac{r}{d}=\frac{m}{r}=\frac{s}{f}=\frac{l}{s}=\frac{t}{l}=\frac{4}{\mathrm{q}^{2}}$ & $\frac{16}{9}$ & 1.7818 & 10 \\
\hline $\operatorname{minor} 2 \mathrm{nd}$ & $\frac{f}{m}=\frac{2 d}{t}=\frac{8}{\mathrm{q}^{5}}$ & $\frac{16}{15}$ & 1.05946 & 1 \\
\hline major 7 th & $\frac{2 m}{f}=\frac{2 d}{t}=\frac{\mathrm{q}^{5}}{4}$ & $\frac{15}{8}$ & 1.8877 & 11 \\
\hline chromatic & $\frac{2 m}{f}=\frac{2 d}{t}=\frac{\mathrm{q}^{5}}{4}$ & $\frac{25}{24}$ & 1.05946 & 1 \\
\hline aug. 4 th & $\frac{t}{f}=\frac{\mathrm{q}^{6}}{8}$ & $\frac{45}{32}$ & 1.4142 & 6 \\
\hline dim. 5th & $\frac{2 f}{t}=\frac{16}{\mathrm{q}^{6}}$ & $\frac{64}{45}$ & 1.4142 & 6 \\
\hline dim. 7th & $\frac{64}{q^{9}}$ & $\frac{128}{75}$ & 1.6818 & 9 \\
\hline
\end{tabular}

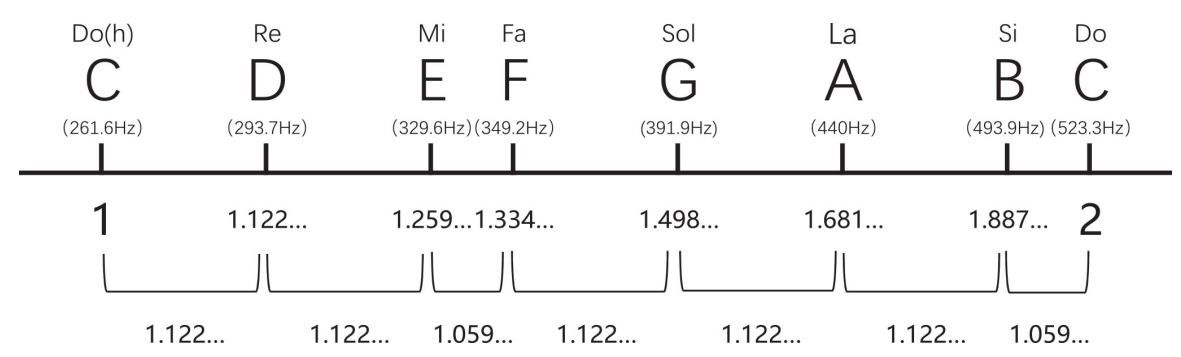

Figure 10. Equal temperament.

collection of 48 preludes and fugues. In this system every semitone is exactly the 12th root of 2 as given in (4.1).

Theorem 9.1 The infinite group of tempered intervals $\left\{\left(2^{1 / 12}\right)^{k} \mid k \in \mathbb{Z}\right\}$ modulo octaves is isomorphic to the cyclic group $\mathbb{Z} / 12 \mathbb{Z}$, i.e.

$$
\left\{\left(2^{1 / 12}\right)^{k} \mid k \in \mathbb{Z}\right\} /\langle 2\rangle \cong \mathbb{Z} / 12 \mathbb{Z} .
$$


The proof follows from the isomorphism theorem on noting that the kernel of the homomorphism

$$
f: \mathbb{Z} \rightarrow\left\{\left(2^{1 / 12}\right)^{k} \mid k \in \mathbb{Z}\right\}
$$

is $12 \mathbb{Z}$.

Example 1 As defined in $\S 1$, the major third consists of 4 semi-tones, and so in equal tempered system it is $\left(2^{1 / 12}\right)^{4}$. The interval from $A\left(f=2^{\frac{7}{12}}\right.$ to $C^{\#} f=2^{\overline{12}} \quad$ being $2^{\frac{4}{12}}$, it is the major third corresponding to $5 / 4$. It is a representative of the equivalence class consisting of the intervals

$$
\left(2^{1 / 12}\right)^{4},\left(2^{1 / 12}\right)^{16},\left(2^{1 / 12}\right)^{8},\left(2^{1 / 12}\right)^{20},\left(2^{1 / 12}\right)^{28}, \cdots
$$

\section{Bach's Fifth}

Recall Corollary 8.2. We consider Kellner's tuning, cf. e.g. [11]. In the major triad $\left\{1, \frac{\mathrm{q}^{4}}{4}\right\}=\{d, m, s\},\{f, l, d\},\{s, t, r\}$, we make q a little smaller than natural 5 th so that the number of beats caused by three times the root and twice the 5 th note be equal to that of the beat caused by the four times the the $3 \mathrm{rd}$ and the 5 th times of the root, i.e. writing such a $\mathrm{q}$ by $\mathrm{q}_{\mathrm{B}}$, we have

$$
3 \times 1-2 \times \mathrm{q}_{\mathrm{B}}=4 \times \frac{\mathrm{q}_{\mathrm{B}}^{4}}{4}-5 \times 1
$$

whence we are led to the Quartic Equation

$$
\mathrm{q}_{\mathrm{B}}^{4}+2 \mathrm{q}_{\mathrm{B}}-8=0
$$

called Bach's equation. Numerically

$$
\mathrm{q}_{\mathrm{B}}=1.495953506 .
$$

\section{Solution of Quintic Equation}

$t$ is well-known that equations with rational (or algebraic) coefficients of degree $\leq 4$ are soluble algebraically, i.e. by ordinary arithmetic operations plus extraction of roots. For our curiosity we shall find the (real) roots of a quintic equation

$$
X^{4}+a X^{2}+b X+c=0
$$

with $a=0, b=2, c=-8$, i.e. (15). This can be solved using its resolvent equation of degree 3:

$$
X^{3}-2 a X^{2}+\left(a^{2}-4 c\right) X+b^{2}=0
$$

which is in the present case

$$
X^{3}-32 X+4=0
$$

with discriminant

$$
D=-4(-32)^{3}-27 \times 4^{2}=16(8192-27)=16 \times 8165
$$

Since $\sqrt{D} \notin \mathbb{Q}$, it follows that the Galois group of $(10.3), \quad X^{4}+2 X-8=0$, is 
the 4th symmetric group $S_{4}$. There exists an explicit formulas for solutions due to Cardano but it is too cumbersome to state. Here we adopt the method of Ohtuska [4], which is indeed Cardano's method. To solve (10.4) one puts

$$
\left\{\begin{array}{l}
u^{3}+v^{3}=-4 \\
3 u v=-32
\end{array}\right.
$$

Then (10.4) becomes

$$
0=X^{3}-3 u v X+u^{3}+v^{3}=(X+u+v)\left(X^{2}+u^{2}+v^{2}+u X+v X+u v\right),
$$

whence $X=-u-v$. On the other hand, (10.5) is easily soluble:

$u^{3}=-2 \pm \sqrt{4+\left(\frac{32}{3}\right)^{3}}$ and we choose $-u=2+\sqrt{4+\left(\frac{32}{3}\right)^{3}}=3.329053233$

At the same time, $-v=2+\sqrt{4-\left(\frac{32}{3}\right)^{3}}=-3.204114179$, so that

$X=-u-v=0.124939054$.

Now recall how the resolvent Equation (10.4) was derived. With $y$ a new variable and $a$ a parameter, we add $2 a X^{2} y+(a y)^{2}$ to both sides of $X^{4}=-b X-c$ with $b=2, c=-8$ to obtain

$$
\left(X^{2}+a y\right)^{2}=2 a y\left(x-2-2 \frac{b}{4 a y} X+\left(\frac{b}{4 a y}\right)^{2}\right)=2 a y\left(\frac{1}{4 a y}\right)^{2},
$$

provided that

$$
a^{2} y^{2}-\frac{b^{2}}{8 a y}-c=0
$$

or

$$
y^{3}-\frac{c}{a^{2}}-\frac{b^{2}}{8 a^{3}}=0, y^{3}+\frac{8}{a^{2}}-\frac{1}{2 a^{3}}=0 .
$$

For this to coincide with the resolvent Equation (10.4) we choose $a=\frac{1}{2}$ and write $-X$ for $y$. Hence (10.6) amounts to

$$
X^{2}+\frac{1}{2} y=-\sqrt{y}\left(X-\frac{1}{y}\right)
$$

whence

$$
X=\frac{1}{2}\left(-\sqrt{y}+\left(\frac{4}{\sqrt{y}}-y\right)^{1 / 2}\right),
$$

which gives the value (10.2) for Bach's fifth.

\section{Music as Duende}

\subsection{Art vs. Technique}

A singer is said to be non-standard original if he or she sings in his or her own original way, not necessarily following exactly the musical notes. The same 
applies to machine music.

The non-standard singer sings a song in his or her original way so that the song is much more appealing to the minds than scholarly way of singing according exactly to the musical notes or machine music.

Thus the music heavily depends on the talent of performers and those non-standard original singers or players are those who are talented enough to grasp the message and intension of the composer to convey it to the audience by expressing it by their "duende".

This class of singers include Hibari Misora, Hideaki Tokunaga, Yumi Matsutoya, or more recent ones, Hikaru Utada, Lia., et al.

It can be speculated that we feel comfortable when we receive stimuli whose frequency are the same as ours, i.e. $1 / f$-noise. This includes the case of music. If it shows $1 / f$-noise, then it gives us comfort.

\subsection{HiFi vs. WiFi}

In ([12], pp. 30-33) the principle of CD is stated. The pitch of the sound can be divided into $2^{16}=65536$ parts because a CD can record 16 convex-concave as one information and transforms into 0 and 1 signal. The $C D$ reads these 16 information 44,100 times per second. The reason for this depends on the assumption that human ears can hear the sound whose frequencies are up to $20 \mathrm{kHz}=20000 \mathrm{~Hz}$. Since the sound with frequency $20,000 \mathrm{~Hz}$ oscillates 20,000 times per second and so more than this times of sampling is needed. And for stereo recording, we need twice as many, whence the sampling frequency 44,100 .

The assumption that all the frequencies higher than $20,000 \mathrm{~Hz}$ may be neglected is rather controversial. For recording simple conversation may not need more, but as supreme art, this omission can be a serious problem because what is missing is often more meaningful as art. We recall that as soon as the real sound is transformed into digital signals, it is not the real signal but an approximation.

Not only in music but flower arrangement, it is said that what is important is rather the space surrounding the arranged flowers. We recall a poem of $\mathrm{R}$. Kinoshita

Peony flowers

So stable and in full bloom

The solidness of the position

The flowers occupy.

\section{Fluctuations}

On fluctuations, there is a bunch of literature. We mention only a few [13] [14] [15] [16] [17]. Let $X(t)$ be a signal and consider its discrete Fourier transform (DFT)

$$
\hat{X}(f)=\sum_{t=1}^{M} \mathrm{e}^{-2 \pi i f t} X(t)
$$


for the frequency $f$. Let the power spectrum be defined by

$$
S_{X}(f)=|\hat{X}(f)|^{2} \text {. }
$$

For a more general treatment of finite power signals, we refer to [1].

It was found (e.g. [15]) that many natural phenomena possess the power spectrum depending on the frequency $1 / f^{\beta}$. This kind of phenomena is called $1 / f$-noise or fluctuation.

In [17], the distribution of primes is considered from the point of view of $1 / f$-fluctuation. However, in [18], there is criticism against the interpretation of power spectrum, saying that it applies to non-living.

We are to study music as something that lies at this threshold of living and non-living.

\section{Conflicts of Interest}

The authors declare no conflicts of interest regarding the publication of this paper.

\section{References}

[1] Takahashi, K., Matsuzaki, T., Hirano, G., Kaida, T., Fujio, M. and Kanemitsu, S. (2014) Fluctuations in Science and Music. Kayanomori, 21, 1-7.

[2] Budden, F.J. (1972) The Fascination of Groups. Cambridge UP, Cambridge.

[3] Coxeter, H.M.S. (1968) Music and Mathematics. The Mathematics Teacher, 61, 312-320.

[4] Ohtsuka, M. (2003) Mathematics of Musical Scores. Waseda Shuppan, Tokyo.

[5] Ouchida, K. (2002) Relationship between Mathematics and Music. Bachelor Thesis, Department of Electrical Engineering, Kinki University, Osaka.

[6] Takahashi, K., Hasegawa, T., Hirano, G., Kaida, T., Kanemitsu, S. and Tsukada, H. (2011) On the Weber-Fechner Law. Siaulai Mathematic Seminar, 6, 85-91.

[7] Adhikari, S.D. and Rath, P. (2008) A Problem in Fractional Parts of the Powers of $3 / 2$ and Related Questions. Proc. Intern. Conf.---Number Theory and Discrete Geometry, Ramanujan Mathematical Society Lect. Note Ser, No. 6, 1-12.

[8] Benary, P. and Bachs, J.S. (2005) Wohltemperiertes Klavier. H \& B, Schneider AG, Aarau.

[9] Bach, J.S. (Dehnhard and Kraus Eds.) (1750) Das wohltemperierte Klavier. Wiener Urtext Edition, Shogakukan, Tokyo.

[10] Kellner, H.A. (1978) Was Bach a Mathematician? English Harpsichord Magazine, 2, 32-36.

[11] Kellner, H.A. (1978) Das wohltemperierte Clavier-Tuning and Musical Structure. English Harpsichord Magazine, 2, 137-140.

[12] Kumagai, N. (2007) Ciphers in the Times of the Web-Challenge of Net Security. Chikuma-Shobo, Tokyo.

[13] Musha, T. (1980) The World of Fluctuations-The Mystery of the $1 / f$-Fluctuation in Nature. Kodansha, Tokyo.

[14] Musha, T. (1994) The Way of Thinking of Fluctuations-Pursuing the Mystery of $1 / f$-Fluctuations. NHK Shuppan, Tokyo. 
[15] Robinson, F.N.H. (1974) Noise and Fluctuations. Oxford UP, Oxford.

[16] Takayasu, H., Ogura, H., Ishida, K., Mitsutani, T. and Aoki, K. (1998) Science of Fluctuations. Morikita-Shuppan, Tokyo.

[17] Wolf, M. (1997) $1 / f$ Noise in the Distribution of Prime Numbers. Physica A, 241, 493-499. https://doi.org/10.1016/S0378-4371(97)00251-3

[18] Matsumoto, G. (1996) Love Actives the Brain. Iwanami-Shoten, Tokyo. 